\title{
Electronic structure at nanocontacts of surface passivated CdSe nanorods with gold clusters
}

\author{
Deepashri Saraf and Anjali Kshirsagar* \\ Department of Physics and Center for Modeling and Simulation, \\ University of Pune, Ganeshkhind, Pune 411007, India
}

\begin{abstract}
We report the effects of variation in length on the electronic structure of CdSe nanorods derived from atomic clusters and passivated by fictitious hydrogen atoms. These nanorods are augmented by attaching gold clusters at both the ends to form a nanodumbbell. The goal is to assess the changes at nanolevel after formation of contacts with gold clusters serving as electrodes and compare the results with experimental observations ${ }^{1}$. Calculations involving nanorods of length $4.6 \AA$ to $116.6 \AA$ are performed using density functional theory implemented within plane-wave basis set. The binding energy per atom saturates for nanorod of length $116.6 \AA$. It is interesting to note that upon attaching gold clusters, the nanorods shorter than $27 \AA$ develop metallicity by means of metal induced gap states (MIGS). Longer nanorods exhibit a nanoscale Schottky barrier emerging at the center. For these nanorods, interfacial region closest to the gold electrodes shows a finite density of states in the gap due to MIGS, which gradually decreases towards the center of the nanorod opening up a finite gap. Bader charge analysis indicates localized charge transfer from metal to semiconductor.
\end{abstract}

\section{INTRODUCTION}

Group II-VI semiconductors possess an ionic character which leads to larger band gaps (BGs) and highly coordinated structures in bulk. This property differentiates them from other compound semiconductors in terms of size and structure dependent aspects at nanoscale. CdSe being the most popular amongst them, due to the reproducibility of its optical absorption and emission properties, has potential applications such as biosensors ${ }^{2}$, displays $s^{3}$ and quantum dot lasers ${ }^{4}$. Due to the unique polar axis of hexagonal wurtzite geometry of these semiconductors, these structures often form more anisotropic shapes ${ }^{5}$. These higher aspect ratio polymorphs, which lead to highly polarised emission, have many applications in optoelectronics ${ }^{6}$. Current synthesis techniques even allow for the diversity of geometric structures such as nanowires, nanobelts, nanotubes and nanorods. In particular, one dimensional structures like nanorods have shown to support a high density of excitons and offer the possibility of enhanced transport of dissociated charge carriers. Shape-controlled synthesis for CdSe nanorods has been reported in last decade ${ }^{\underline{7}-\underline{\underline{9}}}$.

Metal-semiconductor (M-S) interfaces and nanostructured systems are also gathering interest among the science community $1,10-13$ due to their potential applications in developing electronic and optoelectronic devices. The space-charge region in bulk M-S interfaces extends upto few nanometers. It is therefore fascinating to study nanostructures which physically are even less than few nanometers. The electronic properties of nanoscale counterpart of bulk M-S interfaces are yet to be understood completely. From the technological point of view, understanding the properties of such nanocontacts is a major step in the route towards the implementation of semiconductor nanocrystals (as well as molecules) in nanoelectronic device architectures. With the aim of finding answers to the specific issues pertaining to nanoelectronic device architectures, Landman et al studied the M-S nanojunction problem theoretically 14 . They showed induction of subgap states near the $\mathrm{Si}-\mathrm{Al}$ interface, decaying in Si nanowire and development of relatively large Schottky barriers in comparison to bulk. Demechenko and Wang calculated electronic structure of CdSe nanowires, in contact with metallic electrodes of experimentally relevant sizes, by incorporating the electrostatic image potential in atomistic single particle Schrödinger equation ${ }^{15}$. They demonstrated strong nanowire-size-dependence of localized electron-hole states induced by the electrode.

In a scanning tunneling spectroscopy study of goldtipped CdSe nanorods (nanodumbbell), Steiner et al observed a gap similar to that in bare CdSe nanorods near the nanodumbbell center, while subgap structure is found near the M-S nanocontact $\underline{\underline{1}}$. They attributed this behaviour to the formation of subgap interface states that vanished rapidly towards the center of the rod, consistent with theoretical predictions given by Landman et $\mathrm{al}^{14}$. These states lead also to modified Coulomb staircase and in some cases to negative differential conductance on the gold tips. The tunneling spectra can be correlated with the theoretical calculation of density of states (DOS). Theoretical predictions regarding range for decay of metal induced subgap states is about $1 \mathrm{~nm} \frac{14}{4}$ whereas experiments indicate that the subgap states exist even up to $5 \mathrm{~nm}$ from the interface ${ }^{\frac{1}{}}$. The experimental estimates are dictated by spatial resolution and the fact that locality of tunneling spectra holds up to approximately the exciton-Bohr radius of material ${ }^{1}$ $\left(\sim 5.6 \mathrm{~nm}\right.$ for $\left.\mathrm{CdSe}^{16}\right)$. Present work is motivated by this experimental work of Steiner et al.

In present work, we study passivated CdSe nanorods of various lengths in order to see the effect of length variation on charge density profile of the rods. We also 
study their density of states profile upon formation of nanocontacts with gold clusters. We found that our results are qualitatively similar to those of Landman et $a^{14}$ and Steiner et $a^{1}$.

\section{COMPUTATIONAL DETAILS}

Our calculations are based on density functional theory, implemented through Vienna Ab-initio Simulations Package ${ }^{17}$ and are performed employing plane augmented wave ${ }^{18}$ with exchange-correlation energy functional as given by Perdew, Burke and Ernzerhof ${ }^{19}$. The valence electronic configurations for $\mathrm{Cd}$, Se and $\mathrm{Au}$ atoms are, $5 s^{2} 4 d^{10}, 4 s^{2} 4 p^{4}$ and $6 s^{1} 5 d^{10}$ respectively. The cut-off energy used in plane wave expansion is $274.34 \mathrm{eV}$. The self-consistent convergence of energy is set to $10^{-5} \mathrm{eV}$. The calculations are performed only at a single $k$-point, namely the center of the Brillouin zone. Occupation numbers are treated according to the Fermi-Dirac scheme with a broadening of $0.001 \mathrm{eV}$. A sufficiently large unit cell is chosen for the free standing CdSe nanorods so that the minimum distance from the cluster boundary to unit cell boundary was $5 \AA$ in each direction. This vacuum region is even larger $(7 \AA)$ for gold attached CdSe nanorods.

To understand the structural stability, latticedynamical calculations (resulting in phonon DOS) are performed within the framework of self-consistent density functional perturbation theory. Force constant matrices thus obtained are used to generate vibrational frequencies of the system. In order to analyse the bonding between gold cluster and CdSe nanorods, Bader charge analysis method is used based on the algorithm developed by Henkelman et al ${ }^{20-22}$.

\section{RESULTS AND DISCUSSIONS}

\section{A. Cluster derived CdSe nanorods}

The nanorods are generated by relaxing a fragment (viz. $\mathrm{Cd}_{3} \mathrm{Se}_{3}$ ) of bulk wurtzite structure of CdSe crystal in $\left[\begin{array}{llll}1 & 0 & 1 & 0\end{array}\right]$ direction, into its global minimum. This fragment has planar structure and can be considered as the basic building block of the longer nanorods $\left(\mathrm{Cd}_{n} \mathrm{Se}_{n}\right)$. In this cluster, $\mathrm{Cd}$ atoms form an equilateral triangle of side $3.22 \AA$ and Cd-Se bond length is $2.51 \AA$. Further, $\mathrm{Cd}_{6} \mathrm{Se}_{6}$ nanorod is constructed by stacking these six atom rings in chair conformation (See Figure 1 a(ii)). This is the smallest nanorod under consideration. The atoms in this geometry relax in such a manner that intraplanar Cd-Se bond lengths are optimized to $2.60 \AA$, and Cd-Se interplanar bond lengths become $2.82 \AA$. The length of this nanorod is found to be $4.34 \AA$. The
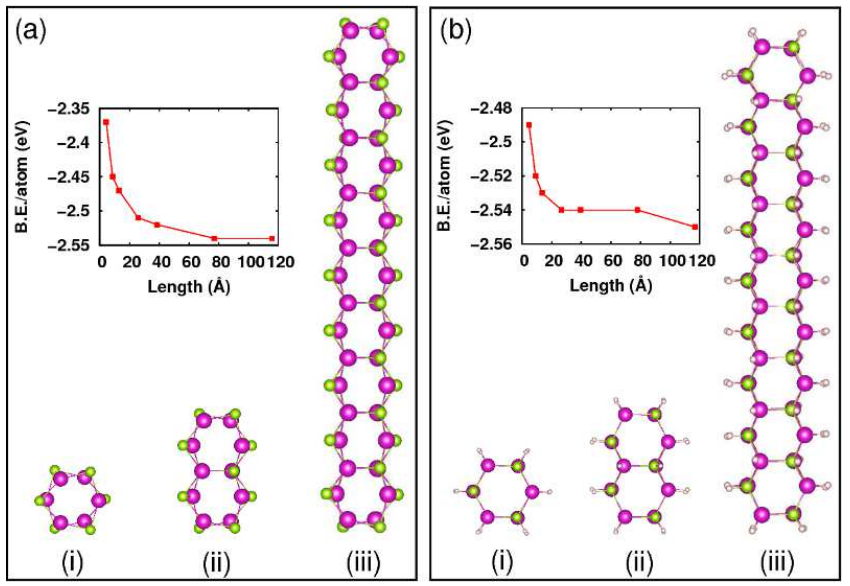

FIG. 1: (color online) Geometries of (a) bare and (b) passivated nanorods of various sizes. (i) $\mathrm{Cd}_{6} \mathrm{Se}_{6}(4.3 \AA, 4.6 \AA)$, (ii) $\mathrm{Cd}_{10} \mathrm{Se}_{10}(8.7 \AA, 9.0 \AA)$ and (iii) $\mathrm{Cd}_{38} \mathrm{Se}_{38}(38.6 \AA$, $39.4 \AA$ ). Numbers in brackets indicate the lengths of the corresponding bare and passivated nanorods respectively. Cd, Se and $\mathrm{H}^{*}$ atoms are indicated in magenta, green and pink respectively. The colour scheme is maintained throughout this article. Insets show the variation of $\mathrm{BE}$ per atom against lengths of the nanorods. Note the difference in the BE per atom scale on $y$-axis in (a) and (b).

longer nanorods are then constructed as the growth over $\mathrm{Cd}_{6} \mathrm{Se}_{6}$ cluster and can be viewed as " $\mathrm{n}$ " $\mathrm{Cd}_{6} \mathrm{Se}_{6}$ clusters coupled together sharing a $\mathrm{Cd}_{2} \mathrm{Se}_{2}$ in common (See Figure 1). Thus, we are dealing with the structures where all the atoms reside on the surface. Binding energy (BE) (defined as the difference between total energies of nanorods and the constituent atoms) indicate that structure is stable. As shown by the graph in the inset of Fig. 1(a), BE per atom reduces initially with the length of the nanorod and then saturates (See also Table II). At smaller lengths of the nanorods, the structures are more or less spherical. But as the length increases the aspect ratio increases, thus making the BE per atom constant.

Surface atoms, due to lower co-ordination number, have incomplete bonding resulting in electronically active states, known as "dangling or unpassivated orbitals". These orbitals are localized and act as efficient traps for charge carriers. At nano-scale, materials become very sensitive to the surface properties due to high surface to volume ratio. Hence, surface passivation is essential for such semiconductor nanostructures. This removes the localized surface states from the band gap. It is experimentally evident that for the II-VI group heteropolar semiconductor nanostructures like CdSe; trioctylphosphine oxide (TOPO) or trioctylphosphine (TOP) are the right choice as passivating agents due to their optimal bonding to the nanostructure surface ${ }^{23-25}$. The complex and large atomic structures of these passivating agents are costly for computational calulations. 
TABLE I: Comparison between the results for passivated and unpassivated nanorods.

\begin{tabular}{|c|cc|cc|cc|}
\hline \multirow{2}{*}{ Structures } & Length $(\AA)$ & $\begin{array}{c}\text { Band gap } \\
(\mathbf{e V})\end{array}$ & \multicolumn{2}{|c|}{$\begin{array}{c}\text { BE per } \\
\text { atom }\end{array}$} \\
\cline { 2 - 7 } & Bare & $+\mathrm{H}^{*}$ & Bare & $+\mathrm{H}^{*}$ & Bare & $+\mathrm{H}^{*}$ \\
\hline \hline $\mathrm{Cd}_{6} \mathrm{Se}_{6}$ & 4.34 & 4.64 & 2.02 & 3.17 & -2.37 & -2.49 \\
$\mathrm{Cd}_{10} \mathrm{Se}_{10}$ & 8.65 & 9.04 & 1.98 & 2.99 & -2.45 & -2.52 \\
$\mathrm{Cd}_{14} \mathrm{Se}_{14}$ & 12.91 & 13.50 & 2.03 & 2.94 & -2.47 & -2.53 \\
$\mathrm{Cd}_{26} \mathrm{Se}_{26}$ & 25.76 & 26.60 & 1.99 & 2.71 & -2.51 & -2.54 \\
$\mathrm{Cd}_{38} \mathrm{Se}_{38}$ & 38.55 & 39.43 & 1.95 & 2.52 & -2.52 & -2.54 \\
$\mathrm{Cd}_{74} \mathrm{Se}_{74}$ & 76.96 & 77.78 & 1.88 & 2.42 & -2.54 & -2.54 \\
$\mathrm{Cd}_{110} \mathrm{Se}_{110}$ & 115.85 & 116.66 & 1.87 & 2.35 & -2.54 & -2.55 \\
\hline
\end{tabular}

Hence, one needs to explore simpler computational methods for surface passivation. There are various techniques developed for such calculations and each one has its own advantages and disadvantages $26-28$. For our calculations we use the technique developed by Huang et $\mathrm{al}^{29}$, where fictitious hydrogen atoms, $\mathrm{H}^{*}$, are chosen to complete the co-ordination of the surface atoms. The pseudopotentials for these pseudo-atoms with appropriate nuclear and valence charge, are generated using the Troullier-Martins prescription ${ }^{30}$. The authors suggest that CdSe dots with a wurtzite structure open a maximum band gap when $\mathrm{H}^{*}$ atoms with valence electron charge $\mathrm{Z}=1.5$ are used to bond with $\mathrm{Cd}$ atoms (cations) and $\mathrm{Z}=0.5$ to bond with Se atoms (anions), thus maintaining the charge neutrality of the whole system. For passivated CdSe nanorods, the wurtzite symmetry is maintained (See Figure 1(b)), but Cd-Se bond length is now elongated upto $2.71 \AA$ and lengths increase slightly (maximum upto $\sim 0.9 \AA$ ) with respect to the non-passivated nanorods. $\mathrm{Cd}$ atoms bond with $\mathrm{H}^{*}$ atoms with bond length $1.84 \AA$ while Se- $\mathrm{H}^{*}$ bond length is $1.60 \AA$. The structural stability of these nanorods is also confirmed using vibrational frequency analysis. Absence of imaginary frequencies establishes that the geometries of these nanorods are locally stable.

Table I gives the results of the calculations for passivated and unpassivated CdSe nanorods of different lengths. BE per atom for passivated structures indicates the same trend as that of non-passivated structures (See inset of Fig. 1(b)), but is lower for passivated structures with low aspect ratio. Thus, passivation takes care of the surface effects prevalent in structures of lower aspect ratios. A comparison of local density of states (LDOS)
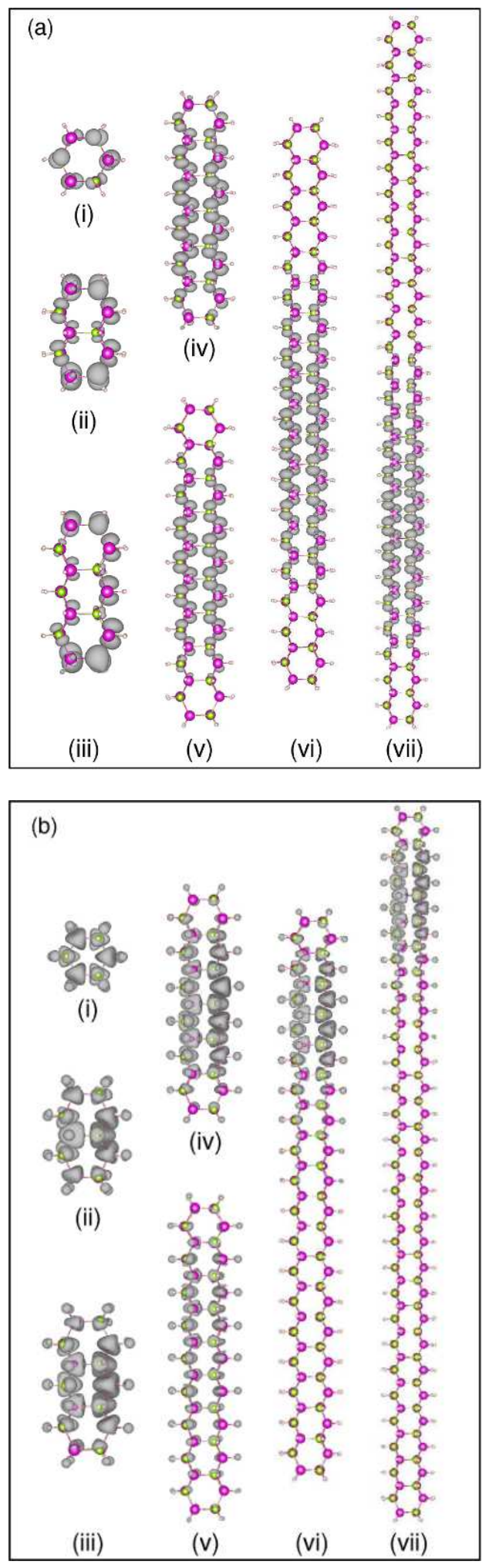

FIG. 2: Partial charge densities of the nanorods showing states HOMO in (a) and LUMO in (b). Note the presence of $p$-states in HOMO on Se atoms while $s$ as well as $p$-states in LUMO on $\mathrm{Cd}$ and Se atoms. As the length of the rods increases, there is a growing asymmetry in the distribution of these states whose span saturates beyond a certain length. 
of these structures also indicates that passivation does not change the intrinsic behavior of highest occupied molecular orbitals (HOMO) and lowest unoccupied molecular orbitals (LUMO). Hence, we could safely conclude that surface passivation is essential in case of such CdSe one dimensional structures. Inspection of BG values of passivated nanorods shows a significant increase by upto $1.1 \mathrm{eV}$ for the smallest length of nanorod. Thus, surface passivation removes the localized surface states from the gap region and these results contradict "self-healing" reported by Puzder et al $\underline{131}$ in their study of CdSe clusters in the form of wurtzite cages of different diameters. In their work, Puzder et al observe that the surface relaxation in CdSe nanostructures act in similar manner to that of passivated nanostructures of CdSe by opening the gap substantially.

Figures 2(a) and (b) show the partial charge density plots for HOMO and LUMO of passivated CdSe nanorods respectively. The partial charge density distribution of HOMO is predominantly localized on Se atoms which comes from the $p_{y}$ orbitals (which lie along the length of the rod) of the atoms while that of LUMO is on both $\mathrm{Cd}$ and Se atoms arising from $s$ orbitals of both $\mathrm{Cd}$ and Se throughout the nanorod. This is also reflected in the site projected DOS (See Figure 3). For short nanorods, $(<15 \AA), p_{z}$ orbitals of Se atoms at the edges also contribute to HOMO. The range of partial charge density arising from HOMO saturates at $39 \AA$ for longer rods. In case of LUMO, as the length of the nanorods increases, the contribution to the partial charge density from $\mathrm{Cd}$ atoms, goes on decreasing. The span of LUMO saturates over the length of $30 \AA$. The passivating agents show the contribution from $s$ orbitals in the LUMO of the nanorods. Bader charge analysis shows that the bonding between $\mathrm{Cd}$ and Se atoms in the nanorods has a partial ionic character with Cd transferring an average charge of $0.55 e$ on Se.

\section{B. Gold tipped CdSe nanorods}

Global minimum geometry of $\mathrm{Au}_{13}$ cluster is found to be suitable to attach at the ends of the nanorods. In this cluster, gold atoms are arranged in three distinct planes. The bare clusters are attached to the fully relaxed nanorods. These compound structures are then optimized fully forming a dumbbell-like structure (nanodumbbell). We observe that the nanorods are unaffected due the end passivation by gold electrodes, except at the M-S nanojunctions, where CdSe bond length elongates to increase the area of the quadrilateral formed by two CdSe pairs. Geometry of gold cluster however changes significantly (See Figures 4(ii) and (iii)). We can observe a slight buckling in three planes of the cluster towards M-S interface. Both $\mathrm{Cd}$ and Se atoms at the ends of the nanorods are doubly bonded to

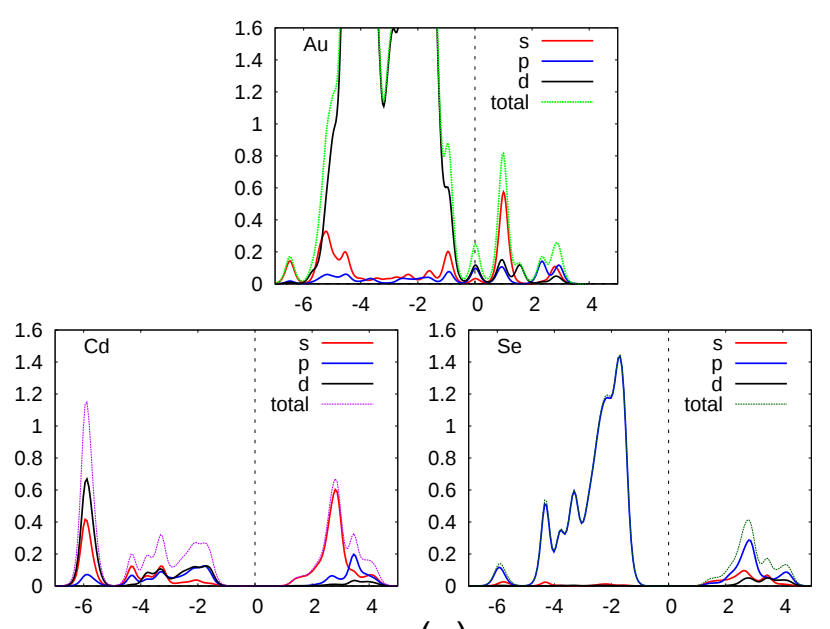

(a)

FIG. 3: Site projected DOS of $\mathrm{Au}$ atom in gold cluster $\left(\mathrm{Au}_{13}\right)$ and $\mathrm{Cd}$ and Se atoms in free standing passivated nanorods $\left(\mathrm{Cd}_{n} \mathrm{Se}_{n}\right)$. Note the dominant presence of $p$ states of Se in HOMO and $s$ and $p$ states of Cd as well as Se in LUMO.

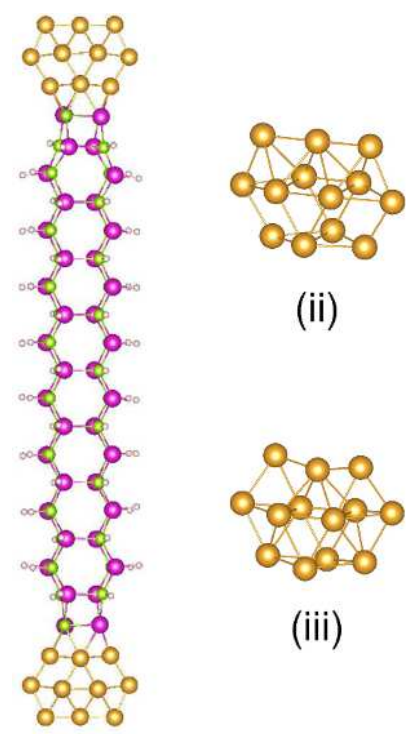

(i)

FIG. 4: (i) Gold $\left(\mathrm{Au}_{13}\right)$ tipped passivated $\mathrm{Cd}_{38} \mathrm{Se}_{38}$ nanorod. $\mathrm{Au}_{13}$ clusters : (ii) bare ; (iii) upon attaching at the tips of CdSe nanorods. Notice the buckling in the geometry upon attaching at the tips of the rods. Gold atoms at the interface are bonded to $\mathrm{Cd}$ as well Se atoms at the tips of the rods.

the $\mathrm{Au}$ atoms at the junctions, where $\mathrm{Au}-\mathrm{Cd}$ and $\mathrm{Au}-\mathrm{Se}$ average bond lengths are $2.74 \AA$ and $2.85 \AA$ respectively.

LDOS calculated for every plane of the gold tipped nanorods (See Figure 5), shows a gap near nanodumbbell center and metal induced gap states (MIGS) emerge near M-S nanocontact. These MIGS vanish rapidly towards 


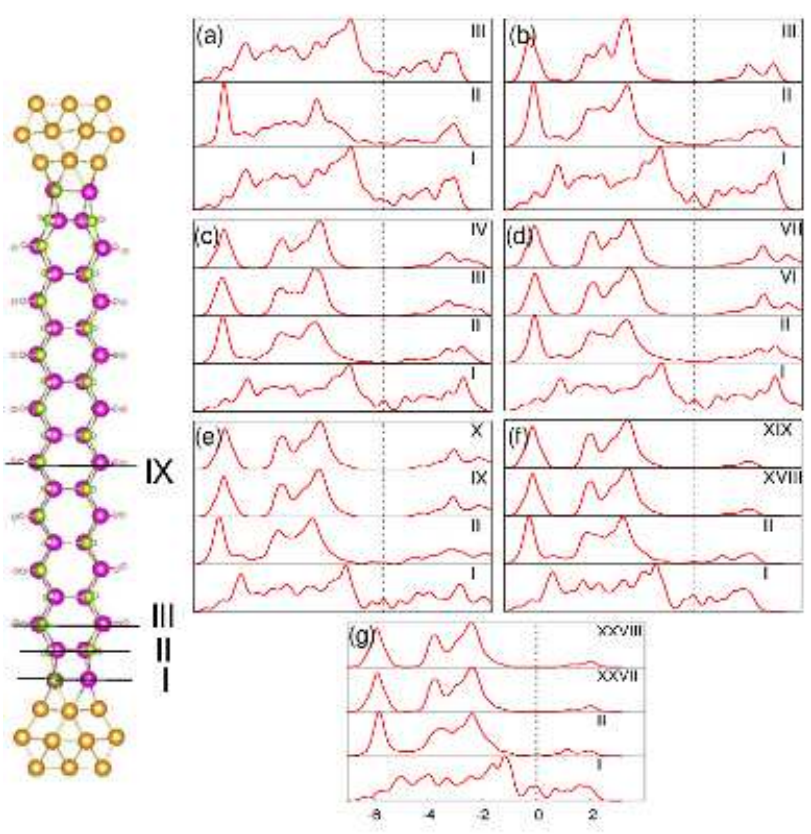

FIG. 5: (color online) LDOS corresponding to various planes, as depicted on the left for gold $\left(\mathrm{Au}_{13}\right)$ tipped passivated $\mathrm{Cd}_{38} \mathrm{Se}_{38}$ nanorod, are shown for following nanorods ( $\mathrm{Au}$ atoms are indicated in yellow): (a) $\mathrm{Cd}_{6} \mathrm{Se}_{6}$, (b) $\mathrm{Cd}_{10} \mathrm{Se}_{10}$, (c) $\mathrm{Cd}_{14} \mathrm{Se}_{14}$, (d) $\mathrm{Cd}_{26} \mathrm{Se}_{26}$, (e) $\mathrm{Cd}_{38} \mathrm{Se}_{38}$, (f) $\mathrm{Cd}_{74} \mathrm{Se}_{74}$ and (g) $\mathrm{Cd}_{110} \mathrm{Se}_{110}$. The Fermi level (shifted to zero of energy) is marked by the vertical dotted line. Each plane in the nanorod contains two CdSe pairs along with their passivating $\mathrm{H}^{*}$ atoms. Numbering is done only up to the central plane owing to the symmetry of the geometry as well as the electronic structure of nanorods (as is evident in (a) for planes I and III). LDOS shows MIGS present at the central plane in structures (a), (b), (c) and (d). These states are absent around the central planes of (e), (f) and (g). The gap between HOMO and LUMO increases as we approach the central plane and saturates for longer rods.

the center of the nanorod 1.14 . For short nanorods (upto $\sim 27 \AA$ ) bridging gold electrodes, we find full metallization by these MIGS (Fig. [5(a), (b), (c) and (d)), while for longer nanorods, we find a gap-structure emerging away from M-S junction. For longer nanorods, in interfacial regions closest to the metal electrodes, a finite LDOS is observed in the gap indicating a metallic nature which gradually decays away from the electrode. A comparison of site projected DOS for gold attached nanorods (See Figure 3) and their separated components, shows the hybridization of metal states with semiconductor states. There is maximum opening of the gap at the central plane of nanodumbbell and the gap size decreases with increasing length of the nanorod, eventually saturating to a value $2.05 \mathrm{eV}$. Figure [6] shows that the plane-wise site projected LDOS for $\mathrm{Au}_{13} \mathrm{Cd}_{38} \mathrm{Se}_{38} \mathrm{Au}_{13}$ nanodumbbell where MIGS start vanishing rapidly across the planes and central plane of
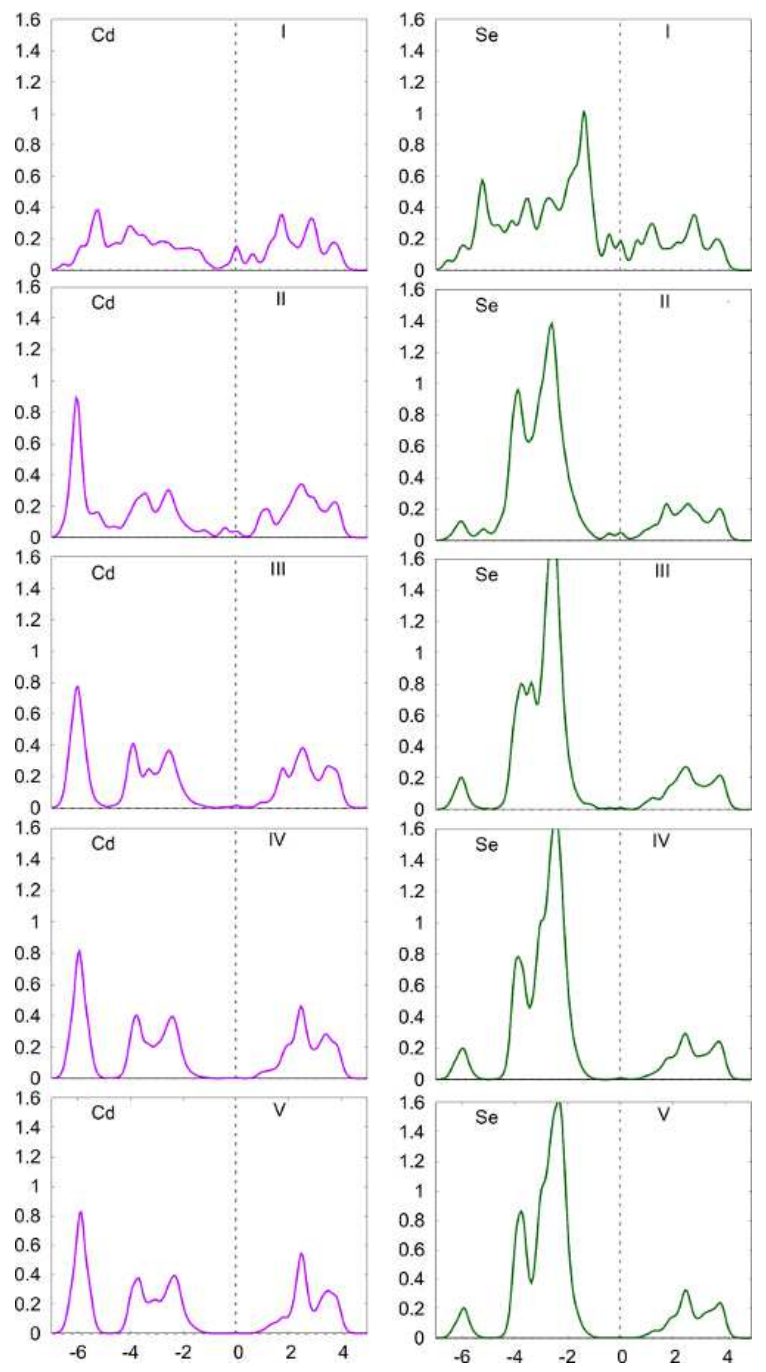

FIG. 6: Plane-wise site projected DOS of Cd atoms in left column and Se atoms in right, in gold tipped $\mathrm{Cd}_{38} \mathrm{Se}_{38}$ rod. In the plane near the junction, one can see the presence of gold states in the gap. These states are also present over a wide energy scale below HOMO, indicating hybridization. Gold states are drastically reduced in the very next plane. As we go towards the center of the rod, the DOS does not show the presence of gold states and the DOS profile of $\mathrm{Cd}$ and Se looks the same as that of free standing nanorod.

the rod shows the presence of only semiconductor states. MIGS are present only upto a distance of $\sim 15.5 \AA$ from the interface, thus making it clear why the nanorods shorter than $27 \AA$ show full metallization.

Figure 7 depicts the partial charge density plots for HOMO and HOMO-1 states for free standing and gold attached passivated $\mathrm{Cd}_{38} \mathrm{Se}_{38}$ nanorod. For the nanodumbbells, the HOMO and HOMO-1 states are comprised of gold $d$ and $s$ states hybridized with $p_{z}$ states of Se and $s$ states of Cd as shown in Fig. [7(b). This behaviour is also evident from the LDOS plots in 

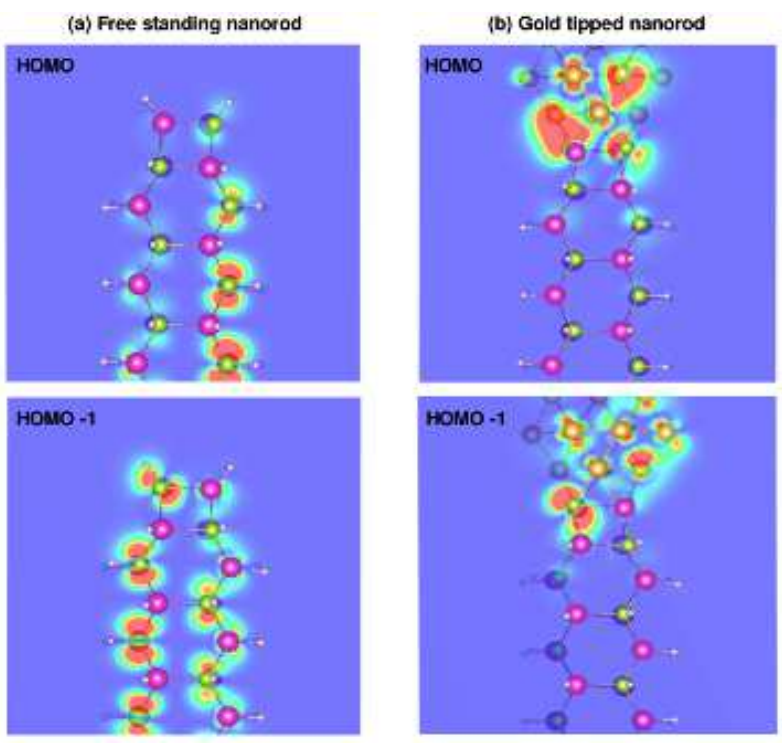

FIG. 7: Partial charge density contour plots for HOMO and HOMO-1 states of (a) free standing and (b) gold tipped passivated $\mathrm{Cd}_{38} \mathrm{Se}_{38}$ nanorod.

TABLE II: Schottky barrier height (SBH) and HOMO-LUMO gap at the central plane as a function of the length of CdSe nanorods. SBH for bulk gold-CdSe contacts is $0.7 \mathrm{eV}$ at $300 \mathrm{~K}^{32}$.

\begin{tabular}{|c|c|c|c|}
\hline Structures & Length & $\begin{array}{c}\text { Schottky barrier } \\
\text { height } \\
(\mathbf{e V})\end{array}$ & $\begin{array}{c}\text { Gap at } \\
\text { central plane } \\
(\mathbf{e V})\end{array}$ \\
\hline \hline $\mathrm{Cd}_{38} \mathrm{Se}_{38}$ & 39.43 & 1.00 & 2.12 \\
$\mathrm{Cd}_{74} \mathrm{Se}_{74}$ & 77.78 & 0.92 & 2.06 \\
$\mathrm{Cd}_{110} \mathrm{Se}_{110}$ & 116.66 & 0.93 & 2.05 \\
\hline
\end{tabular}

Fig. 6. Hybridization of gold states with Cd states is not evident in the partial charge density plots. However, gold induced states appear around $\mathrm{Cd}$ atoms in the contact plane (See Fig. 66 - Plane I and II). The $p_{y}$ states of Se atoms comprising the HOMO and nearby levels in free standing passivated $\mathrm{Cd}_{38} \mathrm{Se}_{38}$ nanorod have shifted below Fermi level $\left(E_{F}\right)$ by $\sim 2 \mathrm{eV}$, while states near HOMO in gold tipped nanorod mainly arise from gold and are spatially localized near the contact plane. Bader charge analysis for the nanodumbbells shows that changes in the charge transfer in comparison to free standing rods are localized near the interface. Bonding remains same away from the interface. At the interface, $\mathrm{Cd}$ atoms transfer an average charge of $0.68 e$ while Se atoms acquire an average charge of $0.53 e$. The remaining charge gets distributed on the gold cluster.
Difference in work function of metal $(\mathrm{Au})$ and electron affinity of semiconductor (CdSe) yields a Schottky barrier when the two are brought in contact. A decrease in the number of available states in confined structures causes an increase in the energy barrier that carriers have to surmount in order to cross the interface. Quantum confinement of carriers is known to increase the minimum energy that a carrier has to have (relative to HOMO for electrons and LUMO for holes) to propagate.

For an n-type semiconductor like CdSe, the Schottky barrier height $(\mathrm{SBH})$ is given by the difference between the metal work function and the electron affinity of the semiconductor. This is calculated from the position of $E_{F}$ in the nanodumbbell and the position of LUMO in the middle section of the CdSe nanorod following Landman et al ${ }^{14}$. The values for SBH are listed in Table III. Group II-VI semiconductors have a higher component of ionic bonding and hence these materials do not create large number of surface states. Their barrier heights therefore depend upon the work function of the metal. Here, the SBH for longer nanorods, which do not show metallization, saturate for the nanorods having lengths longer than $\sim 78 \AA$.

\section{CONCLUSIONS}

In summary, we report that passivation of CdSe nanorods opens up the band gap considerably and we do not observe any "self-healing" as reported by Puzder et $\mathrm{al}^{31}$ for their 3D structures. For free standing nanorods contribution to HOMO comes mainly from $p$ orbitals of Se similar to bulk and 3D confined structures. However contribution to LUMO comes from $s$ orbitals of $\mathrm{Cd}$ and Se while for bulk and 3D structures LUMO mainly consists of $\mathrm{Cd} s$ states. HOMO is confined over a region of $39 \AA$, while LUMO is confined over $30 \AA$ A. Gold attached nanorods are fully metalized for shorter lengths $(<27 \AA)$, while they develop a Schottky barrier, larger than the bulk value $\left(0.7 \mathrm{eV}\right.$ at $\left.300 \mathrm{~K}^{32}\right)$, for longer nanorods, where a semiconducting band gap starts to show up at a distance of $15.5 \AA$ from the nanojunction. End-passivated nanorods and their separated components show charge transfer which is highly localized at contact region.

All the figures of the structures are generated using VESTA $^{33}$. We thank Department of Science \& Technology, Government of India for financial support and C-DAC, Pune for use of their computing facilities. 
Lett. 95, 056805 (2005).

${ }^{2}$ W. C. Chan and S. Nie, Science 281, 2096 (1998).

3 V. L. Colvin, M. C. Schlamp, and A. P. Alivisatos, Nature 370, 354 (1994).

${ }^{4}$ V. I. Klimov, Science 290, 314 (2000).

${ }^{5}$ L. Manna, E. C. Scher, and A. P. Alivisatos, J. Am. Chem. Soc. 122, 12700 (2000).

6 S. A. Empedocles, R. Neuhauser, K. .Shimizu, and M. G. Bawendi, Adv. Mater. 11, 1243 (1999).

7 J. Hu, L. Li, W. Yang, L. Manna, L. Ang, and A. P. Alivisatos, Science 292, 2060 (2001).

8 X. Peng, Adv. Mater. 15, 459 (2003).

9 A. S. Barnard, X. Huifang, L. Xiaochun, N. Pradhan, and X. Peng, Nanotechnology 17, 5707 (2006).

10 R. de Paiva and R. D. Felice, Am. Chem. Soc. NANO 2, 2225 (2008).

11 T. Mokari, C. G. Sztrum, A. Salant, E. Rabani, and U. Banin, Nature Materials 4, 855 (2005).

12 A. Salant, E. Amitay-Sadovsky, and U. Banin, J. Am. Chem. Soc. 128, 10006 (2006).

13 R. Costi, A. E. Saunders, E. Elmalem, A. Salant, and U. Banin, Nano Letters 8, 637 (2008).

14 U. Landman, R. N. Barnett, A. G. Scherbakov, and P. Avouris, Phys. Rev. Lett. 85, 1958 (2000).

15 D. O. Demechenko and L. W. Wang, Nano Lett. 7, 3219 (2007).

16 R. W. Meulenberg, J. R. I. Lee, A. Wolcott, J. Z. Zhang, L. J. Terminello, and T. Buuren, ACS Nano 3, 325 (2009).

17 G. Kresse and J. Furthmuller, Phys. Rev. B 54, 11169
(1996).

18 P. E. Blochl, Phys. Rev. B 50, 17953 (1994).

19 J. P. Perdew, K. Burke, and M. Ernzerhof, Phys. Rev. Lett. 77, 3865 (1996).

${ }^{20}$ W. Tang, E. Sanville, and G. Henkelman, J. Phys.: Condens. Matter 21, 084204 (2009).

21 E. Sanville, S. D. Kenny, R. Smith, and G. Henkelman, J. Comp. Chem. 28, 899 (2007).

${ }^{22}$ G. Henkelman, A. Arnaldsson, and H. Jonsson, Comput. Mater. Sci. 36, 254 (2006).

23 A. P. Alivisatos, Science 271, 933 (1996).

24 J. M. Heath, Acc. Chem. Res. 32, 38 (1999).

25 P. Yang, S. Tretiak, A. E. Masunov, and S. Ivanov, J. Chem. Phys. 129, 074709 (2008).

${ }^{26}$ L. W. Wang and A. Zunger, Phys. Rev. B 53, 9579 (1996).

27 K. Shiraishi, J. Phys. Soc. Jpn. 59, 3455 (1990).

28 J. W. Wang and J. Li, Phys. Rev. B 69, 153302 (2004).

29 X. Huang, E. Lindgren, and J. R. Chelikowsky, Phys. Rev. B 71, 165328 (2005).

30 N. Troullier and J. L. Martins, Phys. Rev. B 43, 1993 (1991).

31 A. Puzder, A. J. Williamson, F. Gygi, and G. Galli, Phys. Rev. Lett. 92, 217401 (2004).

32 S. M. Sze and K. K. Ng, Physics of Semiconductor Devices (John Wiley and Sons, Inc., New Jersey, 2007) page 179.

33 K. Momma and F. Izumi, J. Appl. Crystallogr. 41, 653 (2008). 